\title{
Remarks on the GNS Representation and the Geometry of Quantum States
}

\author{
Dariusz Chruściński \\ Institute of Physics, Nicolaus Copernicus University, \\ Grudziạdzka 5/7, 87-100 Toruń, Poland \\ Giuseppe Marmo \\ Dipartamento di Scienze Fisiche, Universitá "Federico II" di Napoli \\ and Instituto Nazionale di Fisica Nucleare, Sezione di Napoli, \\ Complesso Universitario di Monte Sant Angelo, \\ Via Cintia, I-80126 Napoli, Italy
}

\begin{abstract}
It is shown how to introduce a geometric description of the algebraic approach to the non-relativistic quantum mechanics. It turns out that the GNS representation provides not only symplectic but also Hermitian realization of a 'quantum Poisson algebra'. We discuss alternative Hamiltonian structures emerging out of different GNS representations which provide a natural setting for quantum bi-Hamiltonian systems.
\end{abstract}

\section{Introduction}

The important role played by geometry in the formulation of theories aimed at the descriptions of fundamental interactions cannot be denied. At the moment classical theories like mechanics, electromagnetism, Einstein's General Relativity, Yang-Mills gauge theories and thermodynamics have reached a very high degree of geometrization. The same cannot be said for quantum theories, even though the relevance of geometric structures, like the symplectic structure, may be traced back to Segal and Mackey [1, 2, and since quite few papers have been written on the subject [3-16].

For historical reasons [18 the geometrical structures are hidden in the standard algebraic setting of quantum mechanics (notably the Dirac formulation) because one starts from the Hilbert space and identifies the space of physical states with the associated complex projective space, which in a natural way calls for a differential geometric treatment [4, 5, 8, 10], however, for simplicity, computations are carried out on the initial Hilbert space. In this approach the $\mathbb{C}^{*}$-algebra, which contains observables as real elements, arises as a derived concept - as complex valued functions on the complex projective space endowed with an appropriate associative product even though non-commutative and non-local.

In this short note we would like to consider, on the contrary, a different approach, often called an algebraic one, where the Hilbert space loses its primary importance. The primary 
object one starts with is an abstract $\mathbb{C}^{*}$-algebra containing an algebra of quantum observables and the Hilbert space is a secondary concept which may be derived by constructing particular representation of $\mathcal{A}$ in the spirit of GNS construction (see e.g. [19, 20]). Algebraic approach started with the work of Haag and Kastler [21] and then it was used mainly in the mathematical approach to quantum field theory [22]. This approach is much more flexible than the standard one: a Hilbert space is not a priori given but it is derived by using a given state of the system. Different states give rise to different realization of the original algebra as an algebra of operators, that is, one is able to derive different Hilbert spaces, inner products and multiplication rules in the space of operators acting in the constructed Hilbert space.

The analogy that we pursue is the following: in many classical situations one is presented with a Poisson manifold and looks for a symplectic realization of its Poisson algebra. Here, in a similar way, we would like to consider the 'quantum Poisson algebra' of the complexification of the space of observables and search for a Hermitian realization of it. We observe that this 'Hermitian realization' is the essence of the well known GNS construction.

We start with a $\mathbb{C}^{\star}$-algebra $\mathcal{A}$. It may be decomposed into two real vector spaces of real and imaginary elements

$$
\mathcal{A}_{\mathrm{re}}=\left\{a+a^{*} \mid a \in \mathcal{A}\right\}, \quad \mathcal{A}_{\mathrm{im}}=\left\{a-a^{*} \mid a \in \mathcal{A}\right\},
$$

respectively. There is a one-to-one correspondence between $\mathcal{A}_{\text {re }}$ and $\mathcal{A}_{\text {im }}$ by means of multiplication by ' $i$ '. Consider the space of density states $\mathcal{D}(\mathcal{A})$ over $\mathcal{A}$ which is a convex body spanned by extremal (pure) states $\mathcal{D}^{1}(\mathcal{A})$. Out of the vector space $\mathcal{A}$ we may construct the dual space $L(\mathcal{A})$ by taking real combinations of $\mathcal{D}^{1}(\mathcal{A})$, then we may immerse $\mathcal{A}_{\text {re }}$ into the space of linear functionals on the real vector space $L(\mathcal{A})$. Now, using the commutator product in $\mathcal{A}$ the linear subspace $\mathcal{A}_{\text {re }}$ induces a Poisson structure on $\mathcal{D}(\mathcal{A})$. It shows that the real vector space $L(\mathcal{A})$ constructed out of $\mathcal{D}^{1}(\mathcal{A})$ may be thought of as the dual to $\mathcal{A}_{\text {re }}$ (or equivalently to $\mathcal{A}_{\text {im }}$ ). $L(\mathcal{A})$ may be endowed with a Poisson structure and gives rise to the Lie algebra of Hamiltonian vector fields. It turns out that Hamiltonian vector fields associated with linear maps on $L(\mathcal{A})$ - i.e. element from $\operatorname{Lin}(L(\mathcal{A}), \mathbb{R})$ - may be thought of as derivations of the product available on $\mathcal{A}$ or of the pointwise product that we may construct on the polynomials of $\operatorname{Lin}(L(\mathcal{A}), \mathbb{R})$. The pointwise (commutative) product identifies the Poisson bracket as those of a Poisson algebra (commutative algebra on which derivations act). Moreover, one may introduce a noncommutative $\star$-product in $\operatorname{Lin}(L(\mathcal{A}), \mathbb{C})$, that is, in the $\mathbb{C}^{*}$-algebra of complex valued function on the space of states $L(\mathcal{A}) \supset \mathcal{D}(\mathcal{A})$. In this way the Poisson bracket ' $f \star g-g \star f$ ' with $f, g \in \operatorname{Lin}(L(\mathcal{A}), \mathbb{C})$, should be considered as a quantum bracket in the sense of Dirac. Summarizing: the Poisson bracket on the dual space to $\mathcal{A}_{\text {re }}$ may be used to generate derivations for the commutative algebra of polynomials and therefore as a 'classical' Poisson algebra. The same Poisson bracket when restricted to linear functions defines derivations for the usual (noncommutative) operator product defined in the space of operators but thought of as functions on the dual space. This gives rise to a 'quantum' Poisson algebra.

Having noticed that on the space of self-adjoint elements of a $C^{*}$-algebra one has a Lie algebra structure and a Jordan structure, one may "geometrize", i.e. describe these products in terms of tensorial objects, by using functions and tensors defined on the dual of the $C^{*}$-algebra (Lie algebra). In this way we obtain a Poisson manifold and a Lie-Jordan product associated with a Jordan tensor. 
The main idea for our geometrization uses the dual space of the $C^{*}$-algebra. We recall that in the works of Gelfand and collaborators the dual space of Banach algebras the study of the dual spaces has been found extremely useful. In the sixties Fell [23] has considered dual spaces of $C^{*}$-algebras and Banach algebras providing many interesting results. Here we would like to take a different route and, to present the geometrical ideas more clearly, we restrict to finite dimensional algebras. We shall use, however, an intrinsic formulation, paving the way to an extension to the infinite dimensional situation. We shall use coordinates only to allow the reader to became more familiar with our construction.

The paper is organized as follows: we start with a short review of the geometric formulation of the standard non-relativistic Quantum Mechanics. Then we review GNS construction and provide its simple illustration in the case of matrix algebra in section [5. Section 7 shows how the GNS construction gives rise to the symplectic realization of the Poisson algebra of observables: either via a corresponding Hilbert space defining the representation space of GNS or via the associated complex projective space. In section 8 we discuss alternative Hamiltonian structures emerging out of different GNS realizations of the original $\mathbb{C}^{*}$-algebra. It turns out that the GNS representation provides a natural arena for quantum bi-Hamiltonian systems. Final conclusions are collected in the last section.

\section{Geometric formulation of Quantum Mechanics}

We first review very briefly the geometrical formulation of Quantum Mechanics starting with a standard Hilbert space formulation. The essential steps are the following: The probabilistic interpretation requires that the physical carrier space of our formulation should be identified with the space of rays

$$
\mathbb{C}_{0} \longrightarrow \mathcal{H}_{0} \longrightarrow \mathcal{R}(\mathcal{H})
$$

where $\mathbb{C}_{0}=\mathbb{C}-0$ and $\mathcal{H}_{0}=\mathcal{H}-\{0\}$. The true space of quantum states - space of rays $\mathcal{R}(\mathcal{H})$ - is nothing but the complex projective Hilbert space $\mathbb{P} \mathcal{H}$. Now, to replace vectors and linear transformations by tensor fields we have to replace $\mathcal{H}$ with $T \mathcal{H}$, its tangent bundle, which may be identified as a Cartesian product $T \mathcal{H} \sim \mathcal{H} \times \mathcal{H}$. Any vector $\varphi \in \mathcal{H}$ gives rise to a vector field $X_{\varphi}: \mathcal{H} \longrightarrow T \mathcal{H}$ defined by

$$
X_{\varphi}(\psi):=(\psi, \varphi) \in \mathcal{H} \times \mathcal{H} .
$$

Similarly an endomorphism $A: \mathcal{H} \longrightarrow \mathcal{H}$ gives rise to a map $T_{A}: T \mathcal{H} \longrightarrow T \mathcal{H}$ defined as follows

$$
T_{A}(\psi, \varphi):=(\psi, A \varphi) .
$$

Moreover, one introduces a complex structure $\mathcal{J}: T \mathcal{H} \longrightarrow T \mathcal{H}$ defined by the $(1,1)$-tensor field

$$
\mathcal{J}(\psi, \varphi):=(\psi, i \varphi)
$$

and a linear structure $\Delta: \mathcal{H} \longrightarrow T \mathcal{H}$ defined by the Liouville vector field

$$
\Delta(\psi):=(\psi, \psi),
$$

and finally the so called phase-vector field $\Gamma: \mathcal{H} \longrightarrow T \mathcal{H}$ defined by $\Gamma:=\mathcal{J} \circ \Delta$, i.e.

$$
\Gamma(\psi)=(\psi, i \psi) \text {. }
$$


In this way the Hermitian product $\langle\psi \mid \varphi\rangle$ on $\mathcal{H}$ is replaced by an Hermitian tensor field

$$
\mathcal{K}\left(X_{\varphi_{1}}, X_{\varphi_{2}}\right)(\psi):=\left\langle\varphi_{1} \mid \varphi_{2}\right\rangle
$$

On the corresponding real differential manifold $\mathcal{H}^{\mathrm{R}}$ the real part of $\mathcal{K}$ is a Riemannian metric tensor ' $g$ ' while its imaginary part is a symplectic tensor field ' $\omega$ '

$$
\mathcal{K}=g+i \omega
$$

together with

$$
\omega(X, Y)=g(\mathcal{J} X, Y) .
$$

The above tensor fields endow $\mathcal{H}$ with the structure of a Kähler manifold. When written in a contravariant form $G$ and $\Lambda$, respectively, give rise to two bi-differential operators which may be used to define two brackets on the space of one-forms. We should notice that the symmetric tensor may also be associated with a second order differential operator (Laplacian). These tensor fields may be used to define the metric structure and Poisson bracket on the space of rays $\mathcal{R}(\mathcal{H})$. Note, however, that neither $G$ nor $\Lambda$ can be directly projected from $\mathcal{H}$ to $\mathcal{R}(\mathcal{H})$. It is easy to show that tensor fields which are projectable are given by

$$
\widetilde{G}:=e^{\sigma} G-\Delta \otimes \Delta-\Gamma \otimes \Gamma,
$$

and

$$
\widetilde{\Lambda}:=e^{\sigma} \Lambda-(\Delta \otimes \Gamma-\Gamma \otimes \Delta),
$$

where the conformal factor $e^{\sigma} \geq 0$ is defined by $\sigma(\psi):=\ln \langle\psi \mid \psi\rangle$. Now, projected tensor fields allow for the definition of two products in the space of functions on $\mathcal{R}(\mathcal{H})$ : the symmetric bracket

$$
\left\{f_{1}, f_{2}\right\}_{+}:=\widetilde{G}\left(d f_{1}, d f_{2}\right)+f_{1} \cdot f_{2},
$$

and antisymmetric Poisson bracket

$$
\left\{f_{1}, f_{2}\right\}:=\widetilde{\Lambda}\left(d f_{1}, d f_{2}\right) .
$$

The above operations are defined for arbitrary real valued functions from $\mathcal{F}(\mathcal{R}(\mathcal{H})$ ). In this formulation quantum observables are defined to be functions from $\mathcal{F}(\mathcal{R}(\mathcal{H})$ ) whose Hamiltonian vector fields are at the same time also Killing vector fields, i.e.

$$
\mathcal{F}_{\mathrm{K}}(\mathcal{R}(\mathcal{H})):=\left\{f \in \mathcal{F}(\mathcal{R}(\mathcal{H})) \mid \mathbf{L}_{X_{f}} \widetilde{G}=0\right\}
$$

where $X_{f}=\widetilde{\Lambda}(d f)$. We call such ' $f$ ' a Kählerian function. To deal with complex valued functions, we need the extension from real valued functions to complex valued functions. A complex valued function is Kählerian iff both real and imaginary parts are Kählerian. On this selected space of Kähler functions we may define an associative bilinear product $f \star g$ corresponding to the Hermitian tensor $\widetilde{\mathcal{K}}=\widetilde{G}+i \widetilde{\Lambda}$ :

$$
f \star g:=f \cdot g+\frac{1}{2} \widetilde{\mathcal{K}}(d f, d g)
$$


One shows that for any two Kähler functions ' $f$ ' and ' $g$ ' the nonlocal product ' $f \star g$ ' defines a Kähler function. Consider now the complexified space $\mathcal{F}_{\mathrm{K}}^{\mathbb{C}}(\mathcal{R}(\mathcal{H}))$. Let us observe that any complex valued Kählerian function on $\mathcal{R}(\mathcal{H})$ corresponds to an operator $A \in \mathcal{B}(\mathcal{H})$

$$
A \longrightarrow f_{A}([\psi]):=\frac{\langle\psi \mid A \psi\rangle}{\langle\psi \mid \psi\rangle}
$$

that is, $f_{A}$ is an expectation value function. It is easy to show that

$$
f_{A} \star f_{B}=f_{A B}
$$

Quantum observables correspond to real valued Kählerian functions and hence they are represented by Hermitian operators on $\mathcal{H}$. The complexified space $\mathcal{F}_{\mathrm{K}}^{\mathbb{C}}(\mathcal{R}(\mathcal{H})$ ) equipped with the above noncommutative $\star$-product provides a realization of a $\mathbb{C}^{*}$-algebra corresponding to a $\mathbb{C}^{*}$ algebra of bounded operators acting on the initial Hilbert space $\mathcal{H}$, i.e. the algebra $\mathcal{B}(\mathcal{H})[10$.

Consider now a general Kählerian manifold $(\mathcal{M}, \widetilde{\mathcal{K}})$ not necessarily a projective Hilbert space $\mathbb{P H}$. It is clear that one may define a nonlocal $\star$-product

$$
f \star g:=f \cdot g+\frac{1}{2} \widetilde{\mathcal{K}}(d f, d g)
$$

for arbitrary $f, g \in \mathcal{F}_{\mathrm{K}}^{\mathbb{C}}(\mathcal{M})$. Now, for arbitrary $\mathcal{M}$ the corresponding space of complex valued Kählerian functions is not closed under $\star$-product. The Poisson bracket

$$
\{f, g\}=\frac{i}{2}(f \star g-g \star f),
$$

does belong to $\mathcal{F}_{\mathrm{K}}^{\mathbb{C}}(\mathcal{M})$, however, the symmetric bracket

$$
\{f, g\}_{+}=\frac{1}{2}(f \star g+g \star f),
$$

in general is not a Kählerian function. The condition that the space of Kählerian function over $\mathcal{M}$ is closed with respect to symmetric bracket puts strong conditions on the Kähler structure. It turns out that it is equivalent to the very intricate geometric property of $\mathcal{M}$, namely, that holomorphic sectional curvature of $\mathcal{M}$ is constant [9]. This in turn implies that $\mathcal{M}$ is a projective Hilbert space $\mathbb{P} \mathcal{H}$ or the covering space of the symplectic orbit in $u^{*}(\mathcal{H})$. Thus only orbits of the unitary group are associated with $\mathbb{C}^{*}$-algebras - they will be given by the generating functions of the Hamiltonian action of the unitary group.

After the GNS construction one should be able to prove that realization of the $\mathbb{C}^{*}$-algebras are in one-to-one correspondence with the action of the unitary group on the Kähler manifold.

\section{$3 \quad$ Finite dimensional setting}

Let us illustrate the above geometrical formulation for finite dimensional Hilbert space $\mathcal{H}=$ $\mathbb{C}^{n+1}$. Denote by $\left\{\left|e_{j}\right\rangle\right\}$, with $j=0,1, \ldots, n$, an orthonormal basis in $\mathbb{C}^{n+1}$. Then for any vector $\psi \in \mathbb{C}^{n+1}$ one has

$$
\left\langle e_{j} \mid \psi\right\rangle=z^{j}=: q^{j}+i p^{j}
$$


and

$$
|d \psi\rangle=d z^{j}\left|e_{j}\right\rangle=\left(d q^{j}+i d p^{j}\right)\left|e_{j}\right\rangle .
$$

Using Cartesian coordinate system $\left(q^{j}, p^{k}\right)$ on $\mathcal{H}^{\mathrm{R}}$ one easily finds

$$
\Delta=q^{j} \frac{\partial}{\partial q^{j}}+p^{j} \frac{\partial}{\partial p^{j}},
$$

and

$$
\Gamma=p^{j} \frac{\partial}{\partial q^{j}}-q^{j} \frac{\partial}{\partial p^{j}} .
$$

Moreover, the Hermitian tensor field reads as follows

$$
\begin{aligned}
\mathcal{K}(d \psi, d \psi) & =\overline{\left(d q^{j}+i d p^{j}\right)} \otimes\left(d q^{k}+i d p^{k}\right)\left\langle e_{j} \mid e_{k}\right\rangle \\
& =\left(d q^{j} \otimes d q^{k}+d p^{j} \otimes d p^{k}\right)\left\langle e_{j} \mid e_{k}\right\rangle \\
& +i\left(d q^{j} \otimes d p^{k}-d p^{j} \otimes d q^{k}\right)\left\langle e_{j} \mid e_{k}\right\rangle .
\end{aligned}
$$

The corresponding contravariant tensors $G$ and $\Lambda$ are therefore given by

$$
G=\left(\frac{\partial}{\partial q_{j}} \otimes \frac{\partial}{\partial q_{k}}+\frac{\partial}{\partial p_{j}} \otimes \frac{\partial}{\partial p_{k}}\right)\left\langle e_{j} \mid e_{k}\right\rangle,
$$

and for the Poisson tensor

$$
\Lambda=\left(\frac{\partial}{\partial p_{j}} \otimes \frac{\partial}{\partial q_{k}}-\frac{\partial}{\partial p_{k}} \otimes \frac{\partial}{\partial q_{j}}\right)\left\langle e_{j} \mid e_{k}\right\rangle .
$$

Finally, one may introduce the following local coordinates on $\mathbb{C} P^{n} \equiv \mathbb{P H}$ :

$$
w_{k}=\frac{z_{k}}{z_{0}}
$$

for $z_{0} \neq 0$. Using projective coordinates $\left(w_{1}, \ldots, w_{n}\right)$ one obtains the following formula for $\widetilde{\mathcal{K}}$

$$
\widetilde{\mathcal{K}}=\sum_{i, j=1}^{n} \frac{\left(1+|w|^{2}\right) \delta_{i j}-\bar{w}_{i} w_{j}}{\left(1+|w|^{2}\right)^{2}} d w_{i} \otimes d \bar{w}_{j},
$$

where $|w|^{2}=\sum_{k=1}^{n} w_{k} \bar{w}_{k}$.

Interestingly, Kählerian functions on complex projective space are eigenfunctions of the corresponding Laplacian $\Delta_{n}$. It is well known [24] that the spectrum of the Laplacian on $\mathbb{C} P^{n}$ is given by

$$
\lambda_{n, l}=-l(n+l), \quad l=0,1,2, \ldots,
$$

and the corresponding multiplicity of $\lambda_{n, l}$ reads as follows [25]

$$
N_{n, l}=n(n+2 l)\left(\frac{(n+l-1) !}{n ! l !}\right)^{2}
$$

\footnotetext{
${ }^{1}$ Actually, in 24] the eigenvalues differ by a factor ' 4 '. It corresponds to different normalization of $\Delta_{n}$. Our convention reproduces quantum mechanical result ' $l(l+1)$ ' on $\mathbb{C} P^{1} \cong S^{2}$.
} 
Note that for $l=1$ one obtains

$$
N_{n, 1}=n(n+2)=(n+1)^{2}-1,
$$

that is, it reproduces the dimension of the space of traceless Hermitian operators in $\mathbb{C}^{n+1}$. Now, one may prove that $f$ is Kählerian iff

$$
\Delta_{n} f=0, \quad \text { or } \quad \Delta_{n} f=\lambda_{n, 1} f,
$$

that is, $f$ is either a zero mode of $\Delta_{n}$, or it is an eigenvector of $\Delta_{n}$ corresponding to the first nonvanishing eigenvalue ' $-(n+1)$ '. Since zero mode span 1-dimensional space one finds that the space of Kählerian functions is $(n+1)^{2}$-dimensional, i.e. has the same dimension as the space of Hermitian operators in $\mathbb{C}^{n+1}$.

To see how it works let us consider the simplest case $n=1$. The corresponding projective space $\mathbb{C} P^{1}$ is given by the Bloch sphere $S^{2}$ and the eigenvalue problem $\Delta_{1} f=\lambda_{1,1} f$ is well known from the theory of angular momentum. One has

$$
\Delta_{1} Y_{l m}=-l(l+1) Y_{l m}
$$

where $Y_{l m}$ are spherical harmonics and the integer $m$ runs from $-l$ to $l$. Note, that (3.14) implies that $l=0$ or $l=1$. In the first case $Y_{00}$ defines a constant function on $S^{2}$, whereas in the second case we have three independent dipole functions $Y_{11}=x, Y_{1-1}=y$, and $Y_{10}=z$. A constant function corresponds to the identity operator $\mathbb{I}$. One easily checks that dipole functions correspond to Pauli matrices: $\sigma_{x}, \sigma_{y}$, and $\sigma_{z}$.

\section{Review of the GNS construction}

The geometrization we have presented starts from the Hilbert space formulation of Quantum Mechanics. Now, we would like to consider directly the $\mathbb{C}^{*}$-algebra approach and provide a direct geometrization of this approach. According to the algebraic approach to quantum theory [21, 22, 26] the basic notion is the space of observables which consists of real elements of a $\mathbb{C}^{*}$-algebra with unity $\mathcal{A}$. Note, that observables carry a structure of Jordan algebra equipped with the symmetric Jordan product

$$
a \circ b:=\frac{1}{2}(a b+b a)
$$

and of Lie algebra with the antisymmetric Lie product

$$
[a, b]:=\frac{i}{2}(a b-b a) .
$$

These two products recover an original product in $\mathcal{A}$ :

$$
a b=a \circ b-i[a, b] .
$$

In this approach states are represented by positive, normalized linear functionals on $\mathcal{A}$, that is $\omega \in \mathcal{D}(\mathcal{A})$ (set of states over $\mathcal{A}$ ) if for any $a \in \mathcal{A}$ one has $\omega\left(a a^{*}\right) \geq 0$ and $\omega(\mathbb{1})=1$, where $\mathbb{1}$ 
stands for a unit element in $\mathcal{A}$. That is, the set of states $\mathcal{D}(\mathcal{A})$ may be embedded $\mathcal{D}(\mathcal{A}) \hookrightarrow L(\mathcal{A})$ into the dual of $\mathcal{A}$.

The Hilbert space which in the traditional Schrödinger formalism is considered as a primary object does not any longer play this distinguished role. In the algebraic approach it appears as a secondary object which is constructed out of a selected state of the system under consideration. The construction which associates with each state $\omega$ over $\mathcal{A}$ a particular Hilbert space $\mathcal{H}_{\omega}$ is known as the GNS-construction: note that $\omega$ defines the following pairing between elements from $\mathcal{A}$

$$
\langle a \mid b\rangle_{\omega}=\omega\left(a^{*} b\right) .
$$

Positivity of $\omega$ guarantees that $\langle a \mid a\rangle_{\omega} \geq 0$ but this pairing may be degenerate, that is, one may have $\langle a \mid a\rangle_{\omega}=0$ for $a \neq 0$. To cure this problem one introduces the so called Gelfand ideal $\mathcal{J}_{\omega}$ consisting of all elements $a \in \mathcal{A}$ such that $\omega\left(a^{*} a\right)=0$. The set of classes $\mathcal{A} / \mathcal{J}_{\omega}$ defines a pre-Hilbert space and the positive definite scalar product on $\mathcal{A} / \mathcal{J}_{\omega}$

$$
\left\langle\Psi_{a} \mid \Psi_{b}\right\rangle=\omega\left(a^{*} b\right)
$$

where $\Psi_{a}$ and $\Psi_{b}$ stand for the equivalence classes of $a$ and $b$, respectively:

$$
\Psi_{a}=\left[a+\mathcal{J}_{\omega}\right], \quad \Psi_{b}=\left[b+\mathcal{J}_{\omega}\right] .
$$

Formula (4.5) does not depend on the choice of elements $a$ and $b$ from the classes $\Psi_{a}$ and $\Psi_{b}$. Finally, completing $\mathcal{A} / \mathcal{J}_{\omega}$ in the norm topology induced by the scalar product (4.5) one obtains a Hilbert space $\mathcal{H}_{\omega}$. This construction gives rise to the following representation of $\mathcal{A}$ : for any $a \in \mathcal{A}$ one defines a linear operator $\pi_{\omega}(a)$ acting on $\mathcal{H}_{\omega}$ as follows

$$
\pi_{\omega}(a) \Psi_{b}=\Psi_{a b},
$$

where $b$ is any element from the class $\Psi_{b}$. Moreover, if $\pi_{\omega}$ is a faithful representation (that is, $\left.a \neq 0 \Longrightarrow \pi_{\omega}(a) \neq 0\right)$ then the operator norm of $\pi_{\omega}(a)$ equals the $\mathbb{C}^{*}$-norm of $a$ in $\mathcal{A}$. It is clear that the GNS-construction provides a cyclic representation with a cyclic vector $\Omega \in \mathcal{H}_{\omega}$ corresponding to the class of the unit element in $\mathcal{A}$, i.e. $\Omega=\Psi_{\mathbb{1}}$. Moreover,

$$
\omega(a)=\left\langle\Omega\left|\pi_{\omega}(a)\right| \Omega\right\rangle .
$$

By the duality $\mathcal{A}$ acts on $\mathcal{D}(\mathcal{A})$ and hence a Hilbert space corresponding to a state $\omega \in \mathcal{D}(\mathcal{A})$ is nothing but an orbit of $\mathcal{A}$ passing through $\omega$, i.e. $\mathcal{H}_{\omega} \equiv \mathcal{A} \cdot \omega$.

Note that given any element $b \in \mathcal{A}$ one obtains a new vector $\Psi=\pi_{\omega}(b) \Omega \in \mathcal{H}_{\omega}$. If $\Psi$ has norm one, this defines a new state $\omega_{\Psi}$ over $\mathcal{A}$ given by

$$
\omega_{\Psi}(a)=\left\langle\Psi\left|\pi_{\omega}(a)\right| \Psi\right\rangle
$$

or equivalently

$$
\omega_{\Psi}(a)=\omega\left(b^{*} a b\right),
$$

for all $a \in \mathcal{A}$. One calls states over $\mathcal{A}$ defined by (4.9) vector states of representation $\pi_{\omega}$. More general states may be defined by density operators $\rho$ in $\mathcal{B}\left(\mathcal{H}_{\omega}\right)$ via

$$
\omega_{\rho}(a)=\operatorname{Tr}\left(\rho \pi_{\omega}(a)\right) .
$$


One calls all states (4.11) a folium of the representation $\pi_{\omega}$. Let us recall that two representations $\pi_{1}$ and $\pi_{2}$ of $\mathcal{A}$ defined on two Hilbert spaces $\mathcal{H}_{1}$ and $\mathcal{H}_{2}$, respectively, are equivalent, if there exists a unitary intertwiner $U: \mathcal{H}_{1} \longrightarrow \mathcal{H}_{2}$ such that

$$
U \pi_{1}(a) U^{*}=\pi_{2}(a)
$$

for any $a \in \mathcal{A}$. The GNS representation is universal in the following sense: if $\pi$ is a cyclic representation of $\mathcal{A}$ defined on $\mathcal{H}$, then the vector representation $\omega_{\Psi}$ defined via (4.9) is equivalent to $\pi$ for any normalized $\Psi \in \mathcal{H}_{\omega}$.

Now, a state $\omega$ over $\mathcal{A}$ is pure if and only if it cannot be written as a convex combination of other states from $\mathcal{D}(\mathcal{A})$. It is clear that the set of pure states (denoted by $\mathcal{D}^{1}(\mathcal{A})$ ) defines a set of extremal points of the convex body $\mathcal{D}(\mathcal{A})$. The importance of pure states follows from the following

Theorem 1 A GNS representation $\pi_{\omega}$ of $\mathcal{A}$ is irreducible if and only if $\omega$ is a pure state over $\mathcal{A}$.

\section{Illustration: GNS for matrix algebra}

To illustrate how the Hilbert space emerges out of a $\mathbb{C}^{*}$-algebra $\mathcal{A}$ consider the following simple example. Let $\mathcal{A}=\mathcal{B}\left(\mathbb{C}^{n}\right)$, i.e. the algebra of $n \times n$ complex matrices. Any semi-positive operator $\omega \in \mathcal{B}\left(\mathbb{C}^{n}\right)$ defines a state over $\mathcal{A}$ via

$$
\omega(A)=\operatorname{Tr}(\omega A),
$$

for $A \in \mathcal{A}$. Now, for any $A, B \in \mathcal{A}$ one defines the inner product

$$
\langle A \mid B\rangle_{\omega}=\omega\left(A^{*} B\right)=\operatorname{Tr}\left(B \omega A^{*}\right) .
$$

Let $\omega$ be a rank-1 projector. Then there is a basis $\left\{e_{k}\right\}$ in $\mathbb{C}^{n}$ such that $\omega=\left|e_{1}\right\rangle\left\langle e_{1}\right|$. Hence

$$
\langle A \mid B\rangle_{\omega}=\sum_{k=1}^{n} \bar{A}_{k 1} B_{k 1}=: \sum_{k=1}^{n} \bar{a}_{k} b_{k}
$$

with $a_{k}:=A_{k 1}$ and $b_{k}:=B_{k 1}$. Note, that the corresponding Gelfand ideal is defined as follows:

$$
\mathcal{J}_{\omega}=\left\{X \in \mathcal{A} \mid X_{k 1}=0, k=1, \ldots, n\right\},
$$

that is,

$$
\langle A+X \mid B+Y\rangle_{\omega}=\langle A \mid B\rangle_{\omega},
$$

for any $X, Y \in \mathcal{J}_{\omega}$. This shows that the Hilbert space $\mathcal{H}_{\omega} \equiv \mathcal{A} / \mathcal{J}_{\omega} \subset \mathcal{A}^{*}$ emerging out of rank-1 projector is nothing but $\mathbb{C}^{n}$. It is, therefore, clear that the GNS representation of $\mathcal{A}$ in $\mathcal{H}_{\omega}$ reproduces the defining representation of $\mathcal{B}\left(\mathbb{C}^{n}\right)$. To see that the Hilbert space does indeed depend upon the state over $\mathcal{A}$ consider rank- $m$ projector in $\mathcal{B}\left(\mathbb{C}^{n}\right)$ given by $\omega=p_{1}\left|e_{1}\right\rangle\left\langle e_{1}\right|+$ $\ldots+p_{m}\left|e_{m}\right\rangle\left\langle e_{m}\right|$, with $p_{1}, \ldots, p_{m}>0$ and $p_{1}+\ldots+p_{m}=1$. One obtains

$$
\langle A \mid B\rangle_{\omega}=\sum_{k=1}^{n}\left(p_{1} \bar{A}_{k 1} B_{k 1}+\ldots+p_{m} \bar{A}_{k m} B_{k m}\right)=: \sum_{k=1}^{n}\left(\bar{a}_{k}^{(1)} b_{k}^{(1)}+\ldots+\bar{a}_{k}^{(m)} b_{k}^{(m)}\right)
$$


where

$$
a_{k}^{(j)}=\sqrt{p_{j}} A_{k j}, \quad b_{k}^{(j)}=\sqrt{p_{j}} B_{k j} .
$$

The r.h.s. of (5.6) may be called "normal form" of the Hermitian product. This construction shows very clearly that the Hermitian product on the Hilbert space we have constructed depends on the state. In a sense the "preparation" of the state $\omega$ selects the Hermitian structure in $\mathcal{H}_{\omega}$.

Note that the corresponding Gelfand ideal is defined as follows:

$$
\mathcal{J}_{\omega}=\left\{X \in \mathcal{A} \mid X_{k j}=0, k=1, \ldots, n, j=1, \ldots, m\right\} .
$$

If $m=n$, then $\mathcal{J}_{\omega}$ is trivial. It shows that the resulting Hilbert space reads as $\mathcal{H}_{\omega} \cong \mathbb{C}^{n} \oplus$ $\ldots \oplus \mathbb{C}^{n}$ ( $m$ copies). Now, the corresponding GNS representation $\pi_{\omega}$ is no longer irreducible in $\mathbb{C}^{n} \oplus \ldots \oplus \mathbb{C}^{n}$ but decomposes into the direct sum of $m$ irreducible (defining) representations

$$
\pi_{\omega}=\bigoplus_{k=1}^{m} \pi_{k},
$$

that is $\pi_{\omega}(A)=\mathbb{I}_{m} \otimes A$, where $\mathbb{I}_{m}$ is an $m \times m$ identity matrix.

Let us observe that the form of the inner product (5.2) suggests to define a new multiplication rule in the space of operators in $\mathcal{B}\left(\mathbb{C}^{n}\right)$, indeed from

$$
\langle A \mid B\rangle_{\omega}=\operatorname{Tr}\left(B \omega A^{*}\right),
$$

we may set

$$
A \cdot{ }_{\omega} B:=A \omega B .
$$

It defines a new associative product in $\mathcal{B}\left(\mathbb{C}^{n}\right)$. As we shall see in section 8 this new product turns out to be very useful to define bi-Hamiltonian structure for quantum evolution [27, 28, 29, 30, 31].

\section{Geometrization of algebraic structures}

Let $V$ be a vector space and consider its dual $V^{*}$. One may imbed $V$ into its bi-dual $\left(V^{*}\right)^{*}$

$$
V \ni v \longrightarrow \widehat{v} \in \mathcal{F}\left(V^{*}\right)
$$

by

$$
\widehat{v}(\alpha):=\alpha(v),
$$

for $\alpha \in V$. This imbedding allows to deal with polynomial functions directly associated with multilinear functions on $V^{*} \times \ldots \times V^{*}$ by restricting them to the diagonal, i.e. for any multilinear function

$$
f: V^{*} \times \ldots \times V^{*} \longrightarrow \mathbb{R},
$$

its reduction $\tilde{f}(\alpha):=f(\alpha, \ldots, \alpha)$ is a polynomial function in $\mathcal{F}\left(V^{*}\right)$. Note, that for any $v_{1}, v_{2} \in$ $V$ one defines the product $\widehat{v}_{1} \cdot \widehat{v}_{2}$ by

$$
\left(\widehat{v}_{1} \cdot \widehat{v}_{2}\right)(\alpha):=\widehat{v}_{1}(\alpha) \cdot \widehat{v}_{2}(\alpha),
$$

with $\alpha \in V^{*}$. Clearly, $\widehat{v}_{1} \cdot \widehat{v}_{2}$ defines a polynomial in $\mathcal{F}\left(V^{*}\right)$. 
Suppose now that $V$ carries an additional structure defined by a bilinear operation

$$
B: V \times V \longrightarrow V \text {. }
$$

Let us observe that we may use $B$ to define a 2 -tensor field $\tau_{B}$ by setting

$$
\tau_{B}\left(d \widehat{v}_{1}, d \widehat{v}_{2}\right)(\alpha):=\alpha\left(B\left(v_{1}, v_{2}\right)\right) .
$$

Using

$$
d\left(\widehat{v}_{1} \cdot \widehat{v}_{2}\right)=\left(d \widehat{v}_{1}\right) \cdot \widehat{v}_{2}+\widehat{v}_{1} \cdot\left(d \widehat{v}_{2}\right)
$$

one finds

$$
\tau_{B}\left(d \widehat{v}, d\left(\widehat{v}_{1} \cdot \widehat{v}_{2}\right)\right)=\tau_{B}\left(d \widehat{v}, d \widehat{v}_{1}\right) \cdot \widehat{v}_{2}+\widehat{v}_{1} \cdot \tau_{B}\left(d \widehat{v}, d \widehat{v}_{2}\right),
$$

which shows that $\tau_{B}(d \widehat{v})$ is a derivation of the product (6.4). In this sense we may speak of the geometrical description of the binary product by introducing the tensor field $\tau_{B}$ which defines a bi-differential operator.

Now, we shall consider the special cases when $B$ endows $V$ with the structure of Lie algebra or Jordan algebra. Let us start with a Lie algebra $g=(V, B)$, where $B$ is skew-symmetric and satisfies the Jacobi identity

$$
B\left(v_{1}, B\left(v_{2}, v_{3}\right)\right)+\text { cyclic permutations }=0 .
$$

It is evident that $\Lambda:=\tau_{B}$ defines a Poisson tensor on $\mathcal{F}\left(V^{*}\right)$. Moreover, one may prove that in this case $\Lambda(d \widehat{v})$ is also a derivation of (6.6).

Example: as an example consider the 3-dimensional Lie algebra $V=\mathbb{R}^{3}$ defined by

$$
B\left(v_{1}, v_{2}\right)=a_{3} v_{3}, \quad B\left(v_{2}, v_{3}\right)=a_{1} v_{1}, \quad B\left(v_{3}, v_{1}\right)=a_{2} v_{2},
$$

with $a_{1}, a_{2}, a_{3} \in \mathbb{R}$. Defining 3 coordinate functions

$$
x_{1}=\widehat{v}_{1}, \quad x_{2}=\widehat{v}_{2}, \quad x_{3}=\widehat{v}_{3}
$$

together with

$$
\mathcal{C}\left(x_{1}, x_{2}, x_{3}\right)=\frac{1}{2}\left(a_{1} x_{1}^{2}+a_{2} x_{2}^{2}+a_{3} x_{3}^{2}\right),
$$

one finds for the Poisson tensor

$$
\Lambda=\epsilon_{i j k} \frac{\partial \mathcal{C}}{\partial x_{i}} \frac{\partial}{\partial x_{j}} \wedge \frac{\partial}{\partial x_{k}} .
$$

Note, that $\mathcal{C}$ is a Casimir function, i.e. $\Lambda(\mathcal{C}, f)=0$. By properly choosing $a_{1}, a_{2}, a_{3}$ one obtains all unimodular 3-dimensional Lie algebras.

Consider now $V$ equipped with a Jordan product

$$
B\left(v_{1}, v_{2}\right)=v_{1} \circ v_{2} .
$$

The corresponding Riemann tensor $\mathcal{R}:=\tau_{B}$ is defined by

$$
\mathcal{R}\left(d \widehat{v}_{1}, d \widehat{v}_{2}\right)(\alpha)=\alpha\left(v_{1} \circ v_{2}\right) .
$$


Now, contrary to the Poisson tensor, $\mathcal{R}(d \widehat{v})$ is a derivation of (6.4) but no longer a derivation of the Jordan product (6.14).

Finally, let $(V, \cdot)$ be a $\mathbb{C}^{*}$-algebra. It is equipped both with the antisymmetric Lie product

$$
B\left(v_{1}, v_{2}\right):=\frac{i}{2}\left(v_{1} \cdot v_{2}-v_{2} \cdot v_{1}\right)
$$

and the symmetric Jordan product

$$
B^{\prime}\left(v_{1}, v_{2}\right):=\frac{1}{2}\left(v_{1} \cdot v_{2}+v_{2} \cdot v_{1}\right) .
$$

Let $\Lambda:=\tau_{B}$ and $\mathcal{R}:=\tau_{B^{\prime}}$ be the corresponding Poisson and Riemann tensors. Note, that these two structures endow the real elements of $\mathbb{C}^{*}$-algebra with a structure of a Lie-Jordan algebra

Definition 1 A Lie-Jordan algebra $(\mathcal{B}, \circ,[]$,$) is a real vector space endowed with two bilinear$ operations 'o' and [, ] with the following properties

$$
\begin{aligned}
& a \circ b=b \circ a, \\
& {[a, b]=-[b, a] .}
\end{aligned}
$$

Moreover, Lie-Jordan brackets satisfy the Leibniz rule

$$
[a, b \circ c]=[a, b] \circ c+b \circ[a, c]
$$

and Jacobi identity

$$
[a,[b, c]]=[[a, b], c]+[b,[a, c]]
$$

Finally,

$$
(a \circ b) \circ c-a \circ(b \circ c)=\lambda^{2}[[a, c], b],
$$

for some real number $\lambda$.

Hamiltonian vector fields on $V^{*}$ constructed with $\Lambda$ define derivation for the Jordan product. This construction completes the 'geometrization' of a $\mathbb{C}^{*}$-algebra.

Example: Consider the Lie algebra $u(2)$ in the defining representation on $\mathbb{C}^{2}$. It is spanned by 4 anti-Hermitian matrices $v_{\alpha}=i \sigma_{\alpha}$, with $\alpha=0,1,2,3$, where

$$
\sigma_{0}=\left(\begin{array}{ll}
1 & 0 \\
0 & 1
\end{array}\right), \quad \sigma_{1}=\left(\begin{array}{ll}
0 & 1 \\
1 & 0
\end{array}\right), \quad \sigma_{2}=\left(\begin{array}{cc}
0 & -i \\
i & 0
\end{array}\right), \quad \sigma_{3}=\left(\begin{array}{cc}
1 & 0 \\
0 & -1
\end{array}\right),
$$

are Pauli matrices. Now, let us define coordinate functions

$$
y_{\alpha}(A)=\frac{1}{2} \operatorname{Tr}\left(\sigma_{\alpha} A\right)
$$

for $A \in u(2)$. Using the well known property

$$
\sigma_{k} \sigma_{l}=i \epsilon_{k l m} \sigma_{m},
$$


one obtains the following formulae for the Poisson tensor

$$
\Lambda=2 \sum_{k, l, m=1}^{3} \epsilon_{k l m} y_{k} \frac{\partial}{\partial y_{l}} \wedge \frac{\partial}{\partial y_{m}}
$$

and for the Riemann tensor

$$
\mathcal{R}=\frac{\partial}{\partial y_{0}} \otimes_{s} \sum_{k=1}^{3} y_{k} \frac{\partial}{\partial y_{k}}+y_{0} \sum_{k=1}^{3} \frac{\partial}{\partial y_{k}} \otimes \frac{\partial}{\partial y_{k}},
$$

where $\otimes_{s}$ stands for the symmetrized tensor product, i.e. $a \otimes_{s} b=a \otimes b+b \otimes a$.

Moreover, the Hamiltonian vector fields $H_{\alpha}$ corresponding to coordinate functions $y_{\alpha}$, i.e. $H_{\alpha}=\Lambda\left(y_{\alpha}, \cdot\right)$, are defined as follows

$$
H_{0}=0, \quad H_{k}=\sum_{l, m=1}^{3} \epsilon_{k l m} y_{m} \frac{\partial}{\partial y_{l}}
$$

for $k=1,2,3$. Finally the gradient vector fields $X_{\alpha}$ defined by $X_{\alpha}:=\mathcal{R}\left(y_{\alpha}, \cdot\right)$ read as follows

$$
X_{0}=\sum_{\alpha=0}^{3} y_{\alpha} \frac{\partial}{\partial y_{\alpha}}, \quad X_{k}=y_{k} \frac{\partial}{\partial y_{0}}+y_{0} \frac{\partial}{\partial y_{k}},
$$

for $k=1,2,3$. Note, that

$$
\left[X_{\alpha}, X_{\beta}\right]=y_{\alpha} \frac{\partial}{\partial y_{\beta}}-y_{\beta} \frac{\partial}{\partial y_{\alpha}} .
$$

Finally, one may show that the union of these two distributions $H_{k}$ and $X_{k}(k=1,2,3)$ generates $S L(2, \mathbb{C})$.

\section{Hermitian and Kählerian realizations via GNS construction}

Each pure state $\omega$ over $\mathcal{A}$ gives rise to irreducible representation $\pi_{\omega}$ of $\mathcal{A}$ in the Hilbert space $\mathcal{H}_{\omega}$. It is clear that real elements in $\mathcal{A}$ are represented via $\pi_{\omega}$ by self-adjoint operators in $\mathcal{B}\left(\mathcal{H}_{\omega}\right)$ which are in a one-to-one correspondence with the real Lie algebra $u\left(\mathcal{H}_{\omega}\right)$ of the unitary group $U\left(\mathcal{H}_{\omega}\right)$. The symplectic action of $U\left(\mathcal{H}_{\omega}\right)$ on $\mathcal{H}_{\omega}$ by $(U, \Psi) \longrightarrow U \Psi$, provides us with the corresponding momentum map

$$
\mu_{\omega}: \mathcal{H}_{\omega} \longrightarrow u^{*}\left(\mathcal{H}_{\omega}\right)
$$

where $u^{*}\left(\mathcal{H}_{\omega}\right)$ denotes the dual of the Lie algebra $u\left(\mathcal{H}_{\omega}\right)$. The map is defined by

$$
\mu_{\omega}(\psi)=|\psi\rangle\langle\psi|
$$

Note, that $u^{*}\left(\mathcal{H}_{\omega}\right)$ is a Poisson manifold and hence (7.1) provides a symplectic realization. Let us recall that a symplectic realization of a Poisson manifold $(M, \Lambda)$ is a Poisson map $\Phi: S \longrightarrow M$, where $(S, \Omega)$ is a symplectic manifold. When $S$ is a symplectic vector space one calls $\Phi$ a classical Jordan-Schwinger map [32]. When $S$ is a Hilbert space we shall call it Hermitian realization. 
Now, the action of $U\left(\mathcal{H}_{\omega}\right)$ on $\mathcal{H}_{\omega}$ induces the symplectic action of $U\left(\mathcal{H}_{\omega}\right)$ on the space of rays $\mathcal{R}\left(\mathcal{H}_{\omega}\right)$ via

$$
(U,[\psi]) \longrightarrow[U \psi]
$$

The above action provides us with the corresponding momentum map

$$
\widetilde{\mu}_{\omega}: \mathcal{R}\left(\mathcal{H}_{\omega}\right) \longrightarrow u^{*}\left(\mathcal{H}_{\omega}\right)
$$

defined by

$$
\widetilde{\mu}_{\omega}([\psi])=\frac{\mu_{\omega}(\psi)}{\langle\psi \mid \psi\rangle} .
$$

Now, because the above action preserves also the Riemann tensor, the momentum map relates also this tensor on $\mathcal{R}\left(\mathcal{H}_{\omega}\right)$ with the symmetric tensor on $u^{*}\left(\mathcal{H}_{\omega}\right)$ obtained from the Jordan algebra on $u^{*}\left(\mathcal{H}_{\omega}\right)$. The Hermitian tensor on $\mathcal{R}\left(\mathcal{H}_{\omega}\right)$ will be therefore $\mu_{\omega}$-related to a corresponding tensor on $u^{*}\left(\mathcal{H}_{\omega}\right)$. Again (7.4) provides a symplectic realization. We shall call a symplectic realization $\Phi: S \longrightarrow M$ Kählerian realization if $S$ is a submanifold of the complex projective space. Actually, it was proved by Gromov [46, 47] (see also [48]) that any compact Kählerian manifold may be immersed into the complex projective space (in the analogy to the Whitney theorem about embedding of a manifold into the Euclidean space $\left.\mathbb{R}^{N}\right)$.

The linear structure of $u^{*}\left(\mathcal{H}_{\omega}\right)$ allows for convex combinations in $\mu_{\omega}\left(\mathcal{R}\left(\mathcal{H}_{\omega}\right)\right) \subset u^{*}\left(\mathcal{H}_{\omega}\right)$ and hence enables one to consider density operators. Consider now a general mixed state $\varphi$ over $\mathcal{A}$. The corresponding GNS-representation $\pi_{\varphi}$ is no longer irreducible on $\mathcal{H}_{\varphi}$. One has therefore the following direct sum decomposition

$$
\pi_{\varphi}=\bigoplus_{\alpha} \pi_{\alpha}
$$

where $\pi_{\alpha}$ are irreducible representations of $\mathcal{A}$ on $\mathcal{H}_{\alpha}$, and

$$
\mathcal{H}_{\varphi}=\bigoplus_{\alpha} \mathcal{H}_{\alpha} .
$$

It implies that a 'vacuum' vector $\Omega \in \mathcal{H}_{\varphi}$ decomposes as follows

$$
\Omega=\bigoplus_{\alpha} \Omega_{\alpha}, \quad \Omega_{\alpha} \in \mathcal{H}_{\alpha} .
$$

It is clear that each irreducible representation $\pi_{\alpha}$ corresponds to a pure state $\varphi_{\alpha}$ defined by

$$
\varphi_{\alpha}(a)=\frac{1}{p_{\alpha}}\left\langle\Omega_{\alpha}\left|\pi_{\alpha}(a)\right| \Omega_{\alpha}\right\rangle_{\alpha},
$$

where $\langle\mid\rangle_{\alpha}$ denotes the scalar product in $\mathcal{H}_{\alpha}$, and

$$
p_{\alpha}=\left\langle\Omega_{\alpha} \mid \Omega_{\alpha}\right\rangle_{\alpha} .
$$

Normalization of $\Omega$ implies $\sum_{\alpha} p_{\alpha}=1$. It shows that a mixed state $\varphi$ decomposes as the following convex combination of pure states $\varphi_{\alpha}$

$$
\varphi=\sum_{\alpha} p_{\alpha} \varphi_{\alpha}
$$

that is

$$
\varphi(a)=\sum_{\alpha}\left\langle\Omega_{\alpha}\left|\pi_{\alpha}(a)\right| \Omega_{\alpha}\right\rangle_{\alpha}
$$




\section{Alternative Hamiltonian structures}

We stress that different states over $\mathcal{B}\left(\mathbb{C}^{n}\right)$ give rise to different GNS representations and hence to different realizations of the Hilbert spaces. As we already observed a state over $\mathcal{B}\left(\mathbb{C}^{n}\right)$ corresponds to a positive $n \times n$ matrix $K$ (we replaced abstract $\omega$ by $K$ ) and hence may be used to define an alternative scalar product in $\mathbb{C}^{n}$

$$
z \cdot_{K} w=\sum_{k, l=1}^{n} \bar{z}_{k} K_{k l} w_{l},
$$

for any $z, w \in \mathbb{C}^{n}$. One recovers the standard form if $K=\mathbb{I}$, that is

$$
z \cdot w=\sum_{k=1}^{n} \bar{z}_{k} w_{k} .
$$

Different inner products in $\mathcal{H}$ are associated with different multiplication rules in the space of operators

$$
A \cdot{ }_{K} B=A \cdot K \cdot B,
$$

for any $A, B \in \mathcal{B}\left(\mathbb{C}^{n}\right)$. Note that the product ' $\cdot K$ ' defined by the above formula is associative, and hence $\left(\mathcal{B}\left(\mathbb{C}^{n}\right), \cdot K\right)$ carries a structure of a $\mathbb{C}^{*}$-algebra.

With these alternative associative products we may associate alternative Lie algebra structures and alternative Jordan algebras. According to what we have said earlier they are similar to the alternative Poisson structures we find in classical dynamics when dealing with bi-Hamiltonian systems and complete integrability. To carry the analogy consider now quantum dynamics governed by the Hamiltonian $H$ and suppose, that

$$
[H, K]=H \cdot K-K \cdot H=0 \text {. }
$$

Note that

$$
[A, H]=A \cdot H-H \cdot A=A \cdot_{K} H_{K}-H_{K} \cdot K A=:\left[A, H_{K}\right]_{K},
$$

with

$$
H_{K}=K^{-1} \cdot H \text {. }
$$

It proves that one has two alternative descriptions of quantum evolution: either the standard Heisenberg equation

$$
i \hbar \dot{A}=[A, H],
$$

or the equivalent description using deformed multiplication

$$
i \hbar \dot{A}=\left[A, H_{K}\right]_{K} .
$$

Consider now a description of the quantum systems in terms of the Wigner-Weyl formalism. In this approach an operator $A$ on $\mathcal{H}$ is represented by a function $f_{A}$ on a classical phase space $\mathcal{P}$. The commutative product in the space of functions $\mathcal{F}(\mathcal{P})$ is deformed into the noncommutative $\star$-product such that $f_{A} \star f_{B}=f_{A B}$. Moreover, in the classical limit

$$
\lim _{\hbar \rightarrow 0} \frac{1}{\hbar}\{\{f, g\}\}_{\star}=\{f, g\},
$$


where $\{\{f, g\}\}_{\star}=\frac{1}{2 i}(f \star g-g \star f)$. As was already found by Rubio [45], any associative local product in the commutative algebra of functions $\mathcal{F}(\mathcal{P})$ has the following form

$$
f \cdot k g:=f k g
$$

where $f, k, g \in \mathcal{F}(\mathcal{P})$ and $k>0$. Therefore, one may use this new product ' $k$ ' to define an alternative $\star_{k}$-product

$$
f_{A} \star_{k} f_{B}:=f_{A} \star k \star f_{B}, .
$$

It gives rise to the following equation of motion

$$
i \hbar \dot{f}_{A}=\left\{\left\{f_{A}, f_{H}\right\}\right\}_{\star k},
$$

where the Moyal-like $\star_{k}$ bracket reads as follows

$$
\left\{\left\{f_{A}, f_{B}\right\}\right\}_{\star k}=\frac{1}{2 i}\left(f_{A} \star_{k} f_{B}-f_{B} \star_{k} f_{A}\right) .
$$

Note, that in the 'classical limit'

$$
\lim _{\hbar \rightarrow 0} \frac{1}{\hbar}\left\{\left\{f_{A}, f_{B}\right\}\right\}_{\star k}=k\left\{f_{A}, f_{B}\right\}+f_{A} X_{k}\left(f_{B}\right)-f_{B} X_{k}\left(f_{A}\right),
$$

where $X_{k}$ is a Hamiltonian vector field corresponding to $k$. Interestingly, the 'classical limit' of the Moyal $\star_{k}$ bracket is not a Poisson one but a Jacobi bracket. For $k=1$ one has $X_{k}=0$ and hence one recovers the standard Poisson bracket. Similarly, the 'classical limit' of the symmetric Jordan bracket gives

$$
\lim _{\hbar \rightarrow 0} \frac{1}{2}\left(f_{A} \star_{k} f_{B}+f_{B} \star_{k} f_{A}\right)=f_{A} \cdot k f_{B}
$$

It shows that there are alternative deformation quantization schemes depending upon the associative product $f \cdot_{k} g$ in the original commutative algebra $\mathcal{F}(\mathcal{P})$. The additional function ' $k$ ' has been related to the Kubo-Martin-Schwinger (KMS) state [49, 50].

\section{Conclusions}

The contribution of this paper is to start directly from $\mathbb{C}^{*}$-algebra to 'geometrize' it and then use the GNS construction to recover the Hilbert space. As a matter of fact in our geometric version we naturally obtain a Kähler bundle defined on the space of states. Let us recall

Definition $2 A$ Kähler bundle is a triple $(P, B, p)$, where $P$ (total space) and $B$ (base) are topological spaces and $p: P \longrightarrow B$ is a surjective continuous map. Moreover, for each $b \in B$ the fiber $p^{-1}(b)$ is a Kähler manifold.

Indeed, the space of states over $\mathbb{C}^{*}$-algebra $\mathcal{A}$ is naturally embedded into the dual $L(\mathcal{A})$

$$
e: \mathcal{D}(\mathcal{A}) \longrightarrow L(\mathcal{A})
$$

For any state $\varphi \in \mathcal{D}(\mathcal{A})$ its 'orbit' of $\mathcal{A}$ passing through $\varphi$ defines the Hilbert space $\mathcal{H}_{\varphi}$ with

$$
\langle a \varphi \mid b \varphi\rangle=\varphi\left(a^{*} b\right) .
$$


Now, the embedding (9.1) gives rise to the pull-backed bundle $e^{*}\left(T^{*} \mathcal{A}^{*}\right)$. Its reduction by the left Gelfand ideal $\mathcal{J}_{\varphi}$ at each point provides us with a GNS-bundle which replaces the universal representation of a $\mathbb{C}^{*}$-algebra (as a direct sum of all its irreducible GNS-representations). When $\varphi$ is a pure state we obtain a Kählerian realization of a $\mathbb{C}^{*}$-algebra which generalizes to the quantum setting the symplectic realization of a Poisson manifold.

This bundle turns out to be related to the one defined by Shultz [43] (see also [10]). We shall come back to some of these bundle aspects in a forthcoming paper.

\section{Acknowledgments}

A preliminary account of these results was presented in a series of conferences: Holbaek Quantum Gravity Workshop (May 2008), MATHQCI 2008 CSIC Madrid (March 2008), XII Jornada SIMUMAT: Mathematical Structures of Quantum Mechanics, Geometry and Quanta, Toruń (June 2008). G.M. thanks the organizers of these conferences for inviting him. D.C. thanks Beppe Marmo for the warm hospitality in Naples where the main part of this paper was prepared.

\section{References}

[1] G.W. Mackey, The mathematical foundations of Quantum Mechanics, Benjamin, 1962.

[2] I.E. Segal, Postulates for general quantum mechanics, Ann. Math. 48 (1947) 930-948.

[3] F. Strocchi, Complex coordinates and Quantum Mechanics, Rev. Mod. Phys. 38 (1956) 36-40.

[4] T.W. Kibble, Geometrization of quantum mechanics, Comm. Math. Phys. 65 (1979) 189-201.

[5] V. Cantoni, Geometric aspects of Quantum Systems, Rend. sem. Mat. Fis. Milano 48 (1980) 3542 .

[6] V. Cantoni, Generalized transition probability, Comm. Math. Phys. 44 (1975) 125128.

[7] D.J. Rowe, A. Ryman and G. Rosensteel, Many body Quantum Mechanics as a symplectic dynamical system, Phys. Rev. A 22 (1980) 2362-2372.

[8] R. Cirelli, A. Maniá and L. Pizzocchero, Quantum Mechanics as an infinite dimensional Hamiltonian system with uncertainty structure, J. Math. Phys. 31 (1984) 2891-2903 (part I and II).

[9] R. Cirelli, A. Maniá and L. Pizzocchero, A functional representation for non-commutative $\mathbb{C}^{*}$-algebras, Rev. Math. Phys. 6 (1994) 675-697.

[10] M.C. Abati, R. Cirelli, P. Lanzavecchia and A. Maniá, Pure states of general quantum mechanical systems as a Kähler bundles, Nuovo Cimento B 83 (1984) 43-60.

[11] A. Heslot, Quantum Mechanics as a classical theory, Phys. Rev. D 31 (1985) 1341-1348.

[12] J.S. Anandan, A Geometric approach to Quantum Mechanics, Found. Phys. 21 (1991) $1265-1284$. 
[13] A. Ashtekar and T.A. Schilling, Geometrical formulation of Quantum Mechanics, on Einsteins path, pp. 2365, New York: Springer, 1999.

[14] D. Brody and L.P. Hughston, Geometric Quantum Mechanics, J. Geom. Phys. 38 (2001) 1953.

[15] V.I. Manko, G. Marmo, E.C.G. Sudarshan and F. Zaccaria, The geometry of density states, Rep. Math. Phys. 55 (2005) 405-422.

[16] J. Grabowski, M. Kuś and G. Marmo, Geometry of quantum systems: density states and entanglement, J. Phys. A: Math. Gen 38 (2005) 10217-10244.

[17] J. Grabowski, M. Kuś and G. Marmo, Symmetry, group actions and entanglement, Open sys. \& Inform. Dyn. 13 (2006) 343-362.

[18] G. Esposito, G. Marmo, and E.C.G. Sudarshan, From Classical to Quantum Mechanics: An Introduction to the Formalism, Foundations and Applications, Cambridge University Press, 2004 .

[19] O. Bratteli and D.W. Robinson, Operator algebras and quantum statistical mechanics, Springer-Verlag, Berlin, 1987.

[20] R.V. Kadison and J.R. Ringrose, Fundamentals of the theory of operators algebras, Vol. I \& II, Academic Press, Orlando, 1986.

[21] R. Haag and D. Kastler, An algebraic approach to quantum field theory, J. Math. Phys. 5 (1964) 884-861.

[22] R. Haag, Local quantum physics: fields, particles, algebras, Springer-Verlag, 1992.

[23] J.M.G. Fell, The dual space of $\mathbb{C}^{*}$-algeabras, Trans. Amer. Math. Soc. 94 (1960) 365-403; The dual space of Banach algeabras, Trans. Amer. Math. Soc. 114 (1965) 227-250; $\mathbb{C}^{*}$ algebras with smooth dual, Illinois J. Math. 4 (1960) 221-230; Algebras and fibre bundles, Pacific J. Math. 16 (1966) 497-503.

[24] A. Ikeda, Y. Taniguchi, Spectra and eigenforms of the Laplacian on $S^{n}$ and $\mathbb{C} P^{n}$, Osaka J. Math, 15 (1978) 515-546.

[25] M. Boucetta, Spectra and symmetric eigentensors of the Lichnerowicz Laplacian on $\mathbb{C} P^{n}$, arXiv:0712.2830

[26] J.C. Baez, I.E. Segal and Z. Zhou, Introduction to algebraic and constructive quantum field theory, Princeton University Press, Princeton, 1992.

[27] B. A. Dubrovin, G. Marmo and A. Simoni, Alternative Hamiltonian descriptions for quantum systems, Mod. Phys. Lett. A 5 (1990) 1229-1234.

[28] V.I. Manko, G. Marmo, E.C.G. Sudarshan and F. Zaccaria, Wigners Problem and Alternative Commutation Relations for Quantum Mechanics, Int. J. Mod. Phys. B 11 (1996) 1281-1296. 
[29] J. Cariñena, J. Grabowski and G. Marmo, Quantum Bi-Hamiltonian Systems, Int. J. Mod. Phys. A 15 (2000) 4797-4810.

[30] E. Ercolessi, A. Ibort, G. Marmo and G. Morandi, Alternative Linear Structures Associated with Regular Lagrangians. Weyl quantization and the von Neumann Uniqueness Theorem, math-ph/0602011

[31] E. Ercolessi, A. Ibort, G. Marmo and G. Morandi, Alternative linear structures for classical and quantum systems, to appear in Int. J. Mod. Phys. A.

[32] V.I. Manko, G. Marmo, P. Vitale and F. Zaccaria, A generalization of the Jordan-Schwinger map: classical version and its q-deformatuion, Int. J. Mod. Phys. A 9 (1994) 5541-5561.

[33] S. Kobayashi and K. Nomizu, Foundations of Differential Geometry, Interscience, New York, 1969, Vol II.

[34] N.S. Hawley, Constant holomorphic curvature, Can. J. Math. 5 (1953) 53-56.

[35] J. Igusa, On the structure of a certain class of Kähler varietes, Amer. J. Math. 76 (1954) 669-678.

[36] D. Chruściński and A. Jamiołkowski, Geometric Phases in Classical and Quantum Mechanics, Birkhäuser, Boston, 2004.

[37] B. Simon, Holonomy, the quantum adiabatic theorem, and Berry's phase, Phys. Rev. Lett. 51 (1983) 2167-2170.

[38] Y. Aharonov and J. Anandan, Phase change during a cyclic quantum evolution, Phys. Rev. Lett. 58 (1987) 1593-1596.

[39] Y. Aharonov and J. Anandan, Geometry of quantum evolution, Phys. Rev. Lett. 65 (1990) 1697-1700.

[40] P.A.M. Dirac, The Principles of Quantum Mechanics, Oxford University Press, Oxford 1958.

[41] J. Grabowski and G. Marmo, Binary operations in classical and quantum mechanics, arXiv: math/0201089.

[42] K. McCrimmon, A taste of Jordan algebras, Springer-Verlag, Berlin, 2004.

[43] F.W. Shultz, Pure states as a dual object of $C^{*}$-algebras, Comm. Math. Phys. 82 (1982) 497-502.

[44] E.M. Alfsen and F.W. Shultz, Geometry of state spaces of operator algebras, Birkhäuser, Boston, 2003.

[45] R. Rubio, C.R. Acad. Sc. Paris, 299, Série 1, no. 14 (1984) 699.

[46] M. Gromov, A topological technique for the construction of solutions of differential equations and inequalities, Actes Congrés Inter. Math., (Nice, 1970), Gauthier-Villars, Paris, No 2, $1971,221-225$. 
[47] M. Gromov, Partial differential relations, Springer, Berlin, 1986.

[48] D. Tischler, Closed 2-forms and an embedding theorem for symplectic manifolds, J. Diff. Geom. 12 (1977) 229-235.

[49] H. Basart, M. Flato, A. Lichnerowicz and D. Sternheimer, Deformation theory applied to quantization and statistical mechanics, Lett. Math. Phys. 8 (1984) 483-494.

[50] H. Basart and A. Lichnerowicz, Conformal symplectic geometry, deformations, rigidity and geometrical (KMS) conditions, Lett. Math. Phys. 10 (1985) 167-177. 